\title{
Discussion on Fully Automatic Portable Satellite Communication System
}

\author{
Ting Zhang ${ }^{\mathrm{a},}$, Zuliang Wang ${ }^{\mathrm{b}}$, Youqi Hui \\ Department of Information Engineering, Xijing University,Shanaxi , Ousai Communication Science \\ and Technology, Xi'an, China

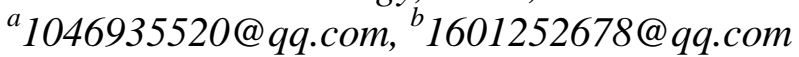

Keywords: fully automatic, portable, star searching, communication link.

Abstract: The technologies of satellite communication, satellite self-tracking, antenna driving control and mechatronics are studied. The automatic positioning, position calculation, automatic search and automatic tracking of satellite antenna in fully automatic portable satellite communication system are improved. A portable satellite communication system is designed, which is simple in structure, can be carried by a single person, easy to operate, automatic star hunting and high precision for satellites. The system can adapt to all kinds of harsh environment, especially when the ground facilities are completely destroyed, improve the speed of emergency handling, and realize the purpose of fast and convenient establishment of two-terminal ground satellite communication links.

\section{Introduction}

With the attention paid to emergencies, anti-terrorism and explosion prevention and natural disaster prevention and disposal, governments and functional departments are required to communicate smoothly, command real-time and smoothly[1]. Portable satellite communication system is preferred because it is not affected by geographical environment and use space, and can be switched on and put into use quickly. There are more products in the market, and the demand is increasing. But there are the following problems. Firstly, the volume and strength need to be improved; secondly, the intelligent degree of system control function is not high; thirdly, the communication bandwidth should be wide enough to ensure large-capacity multimedia transmission in emergency scene.

In order to improve the existing problems of portable satellite communication system and integrate the market demand, the satellite communication technology, satellite self-tracking technology, antenna driving control technology and mechatronics technology are deeply studied. Combining satellite acquisition technology with wireless control board technology, the Beidou/GPS satellite positioning, attitude measurement and adaptive control technology are applied to realize the rapid acquisition and accurate tracking of satellite targets by antenna. The high performance carbon fiber material is used to realize the rapid development of antenna system through overall 
optimization and fine manufacturing technology. It integrates the satellite communication terminal equipment highly, integrates the hardware and software of each equipment, and operates the keyboard of the portable computer to complete the link and parameter setting of the satellite communication link. The satellite communication system has the advantages of simple structure, single person carrying, easy operation, automatic satellite hunting and high precision for satellite, and meets the requirements of maneuverability and real-time. When the ground facilities are completely destroyed, the satellite communication links on both ends of the ground can be established quickly and conveniently, so that the two-way audio-visual emergency communication links can be carried out.

\section{Purpose and significance}

First, confirm that you have the correct template for your paper size. This template has been tailored for output on the A4 paper size. If you are using US letter-sized paper, please close this file and download the Microsoft Word, Letter file. Satellite application industry is an important part of China's strategic emerging industries and a key area of the "Eighth Route Army" of hard science and technology. It is expected that by the end of the 13th Five-Year Plan, hundreds of satellites will be in orbit, forming a relatively complete application satellite system, laying a good foundation for the rapid development of satellite application industry. In the future, it will be a period of high-speed development of satellite application in China. Shaanxi Province has a complete industry chain with a leading position in China. The "13th Five-Year Plan" for the development of satellite application industry in Shaanxi Province has been issued. Xi'an National Civil Aerospace Industry Base also regards satellite application industry as a key pillar industry. Satellite application industry in the world, especially in Europe, the United States, Israel and other developed countries started early, in the late 1980s has been widely used in military, aerospace, civil and other industries, technology is more mature, product types, high degree of standardization. Satellite application industry started relatively late in China, and now it is generally used in national broadcasting, television, meteorology and other large-scale industries. With the gradual improvement of China's communications satellite system, the recent period is the rapid development of China's communications satellite applications, satellite communications are not limited by geographical environment, can provide a large capacity and fast multimedia transmission all day. In recent years, unexpected events and natural disasters have occurred frequently all over the world. The 2008 Wenchuan Earthquake in Sichuan Province occurred frequently. For a long time, the disaster area has been isolated from the outside world, which has brought great obstacles to report the disaster to the outside world, enabling the higher authorities to obtain disaster information in the first time, and timely make the correct rescue organization strategy, resulting in greater losses. Portable satellite communication earth station is one of the main types of satellite communication stations. It is not affected by the geographical environment and the use of space. It can be quickly opened and put into use. The research of portable satellite communication earth station has an urgent need for disaster relief and emergency response. It is of great significance to study fully automatic portable satellite communication system. When ground facilities are completely destroyed, communication links at both ends of the ground can be established quickly and conveniently to provide emergency communication support. In recent years, the research and development of vehicular satellite communication earth station and portable satellite communication system have been carried out at home and abroad. There are many kinds of products sold in the market, and the demand is increasing. But there are the following problems: first, the volume and strength of the system need to be improved; second, the intelligent degree of the system control function is not high, most of the operators are not professionals, therefore, the operation should be simple and practical; third, the 
communication bandwidth should be wide enough to ensure large-capacity multimedia transmission on the emergency scene.

A portable satellite communication system with simple structure, single person carrying, easy operation, automatic star hunting, high precision and high performance is studied and designed. Under the condition that the ground facilities are completely destroyed, and the ground communication condition is inconvenient because of the frequent site change and movement, the ground end-to-end satellite communication links can be established quickly and conveniently to provide communication support, thus the two-way audio-visual emergency communication links can be carried out.

\section{Research contents}

\subsection{Research on wireless control panel for satellite communication antenna control}

The control board has a wireless 433M transceiver module, which can communicate with the main control board wirelessly. The system can be set at different frequencies for wireless communication. The system has low-voltage detection function. When the supply voltage is less than $1.33 \mathrm{~V}$, the system outputs alarm information to the liquid crystal and automatically stops working.

\subsection{Research on antenna angle position detection device}

The antenna angle detection unit structure, the angle position detection sensor and the angular position information processing unit are studied. The antenna angle position detection unit comprises an antenna pitch angle detection unit and an antenna azimuth detection unit. Among them, the antenna pitch angle detection real-time detection of satellite communication antenna pitch angle, antenna azimuth detection real-time detection of satellite communication antenna azimuth. Corner position detection is realized by Hall sensor, which is mounted on the transmission shaft. During the rotation of antenna azimuth, pitch and polarization, the analog voltage at the tap of potentiometer is detected and converted to angle information in proportion. At each boot, the potentiometer is located at the angle of the antenna azimuth, pitch, and the actual mechanical position of the polarization axis. It does not need to find the zero position to shorten the star-seeking time. The output information of the antenna pitch angle detection unit, the antenna azimuth detection unit, the GPS positioning unit and other units are sent to the microprocessor for optimization processing to improve the positioning accuracy.

\subsection{Research on Algorithm and software technology for rapid acquisition of accurate tracking satellites}

The standardization and performance consistency and stability of control software for batch production system are very important to the quality of final antenna products. Therefore, it is necessary to carry out research on software structure, acquisition and tracking satellite algorithm, and to establish global acquisition satellite tracking accuracy and high communication reliability by using BD/GPS and electronic compass positioning. System software, optimize communication parameters, and achieve independent intellectual property standard batch usage software.

\subsection{Research on miniaturization, lightweight and portability technologies}

Small satellite communication earth station is mainly used for emergency relief communication 
support. When some areas are in disasters or where conventional communication cannot cover, the existing communication network is destroyed and unable to establish links. In this case, how to transport small satellite communication earth station to the scene of disaster area is not restricted by region and environment. Establish satellite communication links. Therefore, how to make satellite communication earth station small, portable and easy to carry is an important problem faced by satellite communication earth station. The project focuses on the structure and component parameters of the antenna, including four-lobe antenna, feeder, terminal system integration machine and antenna pedestal structure design, power amplifier, feed, receiver and other components configuration design and antenna transmission structure design.

\subsection{Research on embedded high integration and low power control circuit design}

Embedded microprocessor is widely used in all kinds of control systems. In order to adapt to field transportation and erection, choosing suitable CPU processing chip can reduce weight, volume and power consumption of the system. Therefore, it is necessary to study and design multi-channel, multi-terminal supporting circuit, select low-power peripheral components, such as motors, sensors, command and control devices, design antenna operation control unit, multi-layer circuit board, etc., to adapt to the installation of small structures, the project team will carry out simulation design for the above requirements, and test verification.

\section{Conclusions}

By improving the automatic portable satellite communication system in the aspects of baseband coding, antenna automatic positioning, position calculation, automatic search, and automatic tracking satellite and so on, the speed of emergency handling is increased, and the purpose of establishing the satellite communication links on both ends of the ground quickly and conveniently is realized.

\section{Acknowledgment}

This study was funded by Key Research and Development Plan Project of Shaanxi Provincial Science \& Technology Department (Program No. 2018ZDXM-NY-014).

\section{References}

[1] Kang Rui Xue. Low power design of portable satellite communication terminal [J]. electronicworld, 2018,09:159160.

[2] Wang Jun, Zhou Jichao, Chen Jianping.Design and Application of Portable Support Equipment

Based on Satellite Communication[J].Digital Technology and Application, 2018,3606:189-190+192.

[3] Li Yang. Research and Design of Large Data Acquisition System for Portable Satellite Earth Station [D]. Nanjing University of Posts and Telecommunications, 2017.

[4] Wang Peng. Research and design of satellite assistant system for portable satellite communication earth station [D]. Nanjing University of Posts and Telecommunications, 2017.

[5] Xia Xue ting. Portable satellite earth station remote service system design [D]. Nanjing University of Posts and Telecommunications, 2017.

[6] Liu Yao. Research and design of high availability of portable satellite communication earth station [D]. Nanjing University of Posts andTelecommunications, 2017.

[7] Ma Shuhui. Research and Design of Portable Radio Frequency Front-end Equipment Monitoring System for Satellite Communication [D]. Nanjing University of Posts and Telecommunications, 2017.

[8] Fortunately early snow, she Chenchen Dynasty. Fire Forces Portable Satellite Station Communication Service Terminal Integration Research [J].Network Security Technology and Applications, 2017,10:142-145.

[9] Pan Lingyun. Portable satellite earth station test system design and implementation [D]. Nanjing University of Posts and Telecommunications, 2017. 
[10] Liu Lihong, Liu Fengkai. Development Review of Portable Satellite Communication Earth Station [J].Radio Engineering, 2017,4708:62-66.

[11] Lu Tian Yu. Research on hardware in the loop simulation of spatial information network routing protocol based on SDN [D]. Nanjing University, 2018.

[12] Zhang Han. Performance of Hypertext Transfer Protocol in satellite networks [D]. Nanjing University, 2018. 